\title{
Recent Progress of Fiber Sensing Technologies in Tianjin University
}

\author{
Tiegen LIU, Kun LIU, Junfeng JIANG, Enbang LI, Hongxia ZHANG, Dagong JIA, \\ and Yimo ZHANG
}

\author{
Key Laboratory of Opto-electronics Information and Technical Science, College of Precision Instrument \& \\ Opto-electronics Engineering, Tianjin University, Tianjin 300072, China \\ *Corresponding author: Tiegen LIU E-mail: tgliu@tju.edu.cn
}

\begin{abstract}
The up to date progress of fiber sensing technologies in Tianjin University are proposed in this paper. Fiber-optic temperature sensor based on the interference of selective higher-order modes in circular optical fiber is developed. Parallel demodulation for extrinsic Fabry-Perot interferometer (EFPI) and fiber Bragg grating (FBG) sensors is realized based on white light interference. Gas concentration detection is realized based on intra-cavity fiber laser spectroscopy. Polarization maintaining fiber (PMF) is used for distributed position or displacement sensing. Based on the before work and results, we gained National Basic Research Program of China on optical fiber sensing technology and will develop further investigation in this area.
\end{abstract}

Keywords: Fiber sensing, high temperature, parallel demodulation, gas detection, distributed sensing

\section{Introduction}

Relying on national-level key disciplines "Optical Engineering", the Key Laboratory of Opto-electronics Information and Technical Science (Tianjin University), Ministry of Education, has received a series of national research projects and gained large experimental platform and equipments worth of 35 million yuan. The researches of Tianjin University are always focused on fiber optical sensing technology and the related detection technologies for many years. And we have received remarkable results in the field of high temperature sensor [1, 2], parallel demodulation of extrinsic Fabry-Perot interferometer (EFPI) and fiber Bragg grating (FBG) sensors [3, 4], gas concentration sensing technology based on intra-cavity $[5,6]$, distributed sensing based on polarization maintaining fiber (PMF) [7-10] and so on. Tianjin University has taken on Natural Science Fund of China, 863 project, major programme of National Development Planning Commission, large project of Tianjin, and other provincial or ministerial funds for more than twenty research projects. We have gained several Scientific and Technological Progress Awards at provincial or ministerial level, and have a number of national patents. Based on the previous work and results, Tianjin University gained National Basic Research Program of China (973 Program) for optical fiber sensing technology. We will focus on the research of gas pressure sensing and biologic optical sensing based on optofluidics theory.

Received: 26 September 2010 / Revised version: 20 October 2010

C The Authors(s) 2010.This article is published with open access at Springerlink.com 


\section{High temperature sensor}

Fiber-optic temperature sensors offer unique advantages. Therefore, optical fiber sensors have been widely adopted and applied in different areas for temperature measurements. Those based on FBG technique have attracted much attention [11, 12], but the fabrication process is rather complex and the grating is fragile. A fiber-optic temperature sensor with extremely simple structure was presented in Tianjin University [1, 2], based on the interference of selective high-order modes in circular optical fibers. When a single-mode fiber (SMF) is connected to a multi-mode fiber (MMF), different modes can be excited in the MMF and supported by the MMF. The relative wavelength variation caused by temperature change can be expressed as

$$
\begin{aligned}
\frac{\Delta \lambda}{\lambda} & =\frac{\partial n}{n \partial T} \Delta T+2 \frac{\partial a}{a \partial T} \Delta T-\frac{\partial L}{L \partial T} \Delta T \\
& =(\alpha+\xi) \Delta T
\end{aligned}
$$

where $n$ is the refractive index, $a$ is the core radius, $L$ is the length of MMF, $\alpha$ and $\xi$ are the coefficient of thermal expansion and the thermo-optic coefficient of MMF material respectively. The temperature can be measured by detecting the wavelength at which a maximum or minimum interference occurs between selective higher-order modes.

A schematic diagram showing the structure of the temperature sensor is depicted in the inset of Fig. 1. The sensor was fabricated by connecting a $50 \mathrm{~mm}$ long MMF with a core radius of $52.5 \mu \mathrm{m}$ and a cladding radius of $62.5 \mu \mathrm{m}$ to a standard communications SMF by fusion splicing. In order to make a single-ended sensor, the free end of MMF was cleaved to achieve a surface perpendicular to the fiber axis. This end surface served as a mirror reflecting the light reaching that surface back to the MMF. Among the modes excited in MMF, the $\mathrm{LP}_{06}$ and $\mathrm{LP}_{07}$ modes have the largest coupling coefficients. At the interface between SMF and MMF, the modes in MMF are coupled to $\mathrm{LP}_{01}$ mode in SMF.

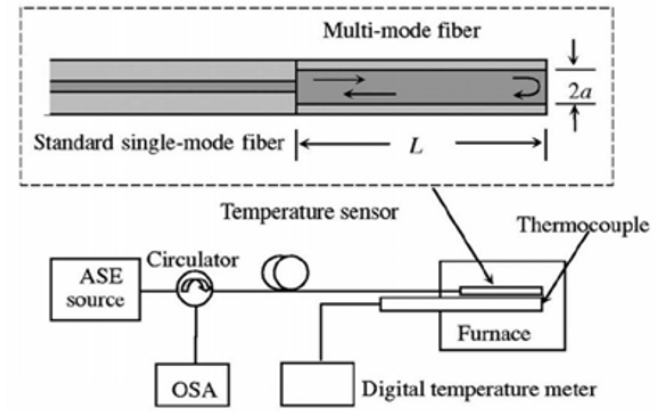

Fig. 1 Schematic diagram of the experimental setup. The inset shows the structure and parameters of the developed temperature sensor.

In order to test the ability and performance of fiber sensor for temperature measurements, the fabricated sensor was attached to a thermocouple, which was connected to a digital temperature meter, and inserted them into a furnace. The measured wavelength shifts under different temperatures are shown in Fig. 2. It can be seen from the experimental data that the wavelength shift varies linearly with temperature changes in the range of $100{ }^{\circ} \mathrm{C}$ to $750{ }^{\circ} \mathrm{C}$ with an average temperature sensitivity of $15 \mathrm{pm} /{ }^{\circ} \mathrm{C}$, which is similar to the temperature sensitivity of the surface relief FBG sensor.

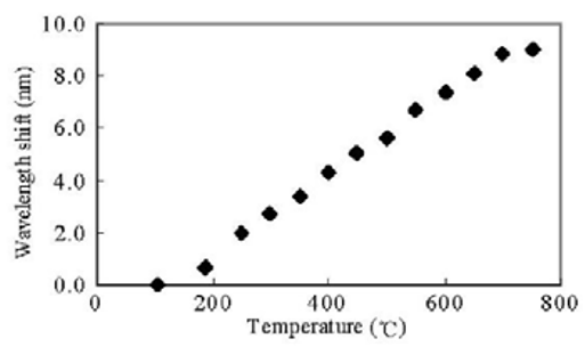

Fig. 2 Measured wavelength shifts under different temperatures.

Compared to other fiber-optic temperature sensors, this sensor features an extremely simple structure and fabrication process, and hence a very low cost. Moreover, the sensors have the potential for high-temperature applications. With the current design, a cleaved surface was used as a reflection mirror, which provided very low reflection. In order to increase the reflectivity, we are now testing fiber mirrors formed by coating aluminum on the surface 
of MMF end.

\section{Parallel demodulation of EFPI and FBG}

Structure health monitoring systems have attracted widespread attention and have been applied to many industrial fields. Since two-parameter or multi-parameter measurement, such as simultaneous strain and temperature measurement, is demanded by structure health monitoring, some combinations of optical fiber sensors make use of the difference in optical fiber sensors' sensitivity to different measurands to construct a sensing matrix and then obtain the measurands through inverse calculation.

A parallel demodulation system for extrinsic EFPI and FBG sensors was presented based on a Michelson interferometer and combined the methods of low coherence interference and Fourier transform spectrum $[3,4]$. The experimental setup is shown in Fig. 3. An amplified spontaneous emission light source with a wavelength covering $1525 \mathrm{~nm}$ to $1610 \mathrm{~nm}$ and $10 \mathrm{~mW}$ of output power is used as a broadband source. A piezo linear motor stage is used as optical path difference (OPD) scanning stage. A retroreflector prism is installed at the scanning stage as an interference arm of Michelson interferometer. A measurement and control platform based on a personal computer can control the movement of the scanning stage and record the interferogram.

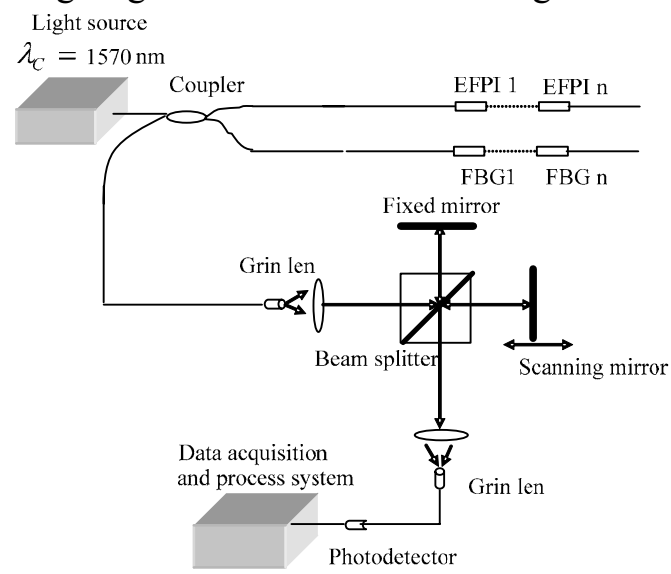

Fig. 3 Parallel demodulation system of an EFPI and a FBG sensor: GRIN(gradient-index).
There exists Fourier relationship between light source spectrum $S(k)$ and the ac term of its interferogram $I(\Delta)$ :

$$
S(k)=\int_{-\infty}^{\infty} I(\Delta) \cos (2 \pi k \Delta) d \Delta
$$

where $k$ is the wave number of light source, $\Delta$ is OPD. When optical signal from FBG array is regarded as light source $S(k)$, the wavelength of FBG can be obtained through fast Fourier transform (FFT) transform of interferogram.

The output of low finesse EFPI can be regarded as two-beam interference. Its output from an ideal Fourier transform spectrometer can be expressed as [13]

$$
\begin{aligned}
& I^{\prime}(\Delta)=2(p+q) g(\Delta) \cos \left(2 \pi k_{0} \Delta\right) \\
& +2 \sqrt{p q}\left[g(\Delta) \cos \left(2 \pi k_{0} \Delta\right)\right] \otimes\left[\delta\left(\Delta-\Delta_{s}\right)+\delta\left(\Delta+\Delta_{s}\right)\right]
\end{aligned}
$$

where $2 g(\Delta) \cos \left(2 \pi k_{0} \Delta\right)$ is the ac term of a broadband source. It shows that three similar interference fringe packets will exist in the output of Fourier transform spectrometer and center on $\Delta=0$, $\Delta=\Delta_{s}, \quad \Delta=-\Delta_{s}$ respectively. Three centers are also peaks of interference fringe packets. Thus the distance between peaks gives the information of EFPI cavity length.

Signals from EFPI and FBG sensors are obtained simultaneously by scanning one arm of Michelson interferometer, and Fourier transform spectrum is used to process the signals and retrieve both the wavelength of FBG and the cavity length of EFPI at the same time as shown in Fig. 4. Fourier transform spectrum of whole interferogram is the overlapping spectrum. The overlapping spectrum region is limited to a small range. So the rest of the spectrum caused only by EFPI, can be used for the cavity length calculation. The Fourier transform of the local interferogram yields the wavelength of FBG. The experiment showed that the demodulation system worked well with measurement errors of $14.4 \mathrm{pm}$ for FBG wavelength and of $0.81 \mathrm{~mm}$ for EFPI cavity. 


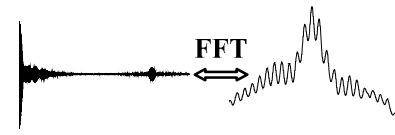

(a)

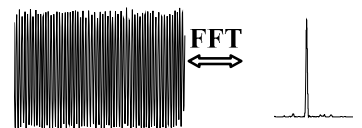

(b)

$\begin{array}{rr}\text { Spatial } & \begin{array}{c}\text { Spectrum } \\ \text { domain }\end{array}\end{array}$

Fig. 4 Interference fringes in spatial domain and corresponding spectrum character in spectrum domain: (a) EFPI and (b) FBG..

The wavelength of FBG and the cavity length of EFPI can be used to determine the strain and temperature. Thus multiparameter detection is realized in this system at the same time.

\section{Gas concentration sensing based on intra-cavity}

Detection of pollution gas is important in environmental and pollution monitoring, which can be used widely in mining and petrochemical industry. Fiber optical spectrum absorption at near-infrared (NIR) wavelength is widely used for gas detection due to its essential advantages. There are several methods, among which intra-cavity laser spectroscopy is a novel way for gas detection.

The schematic diagram of a gas sensing system based on intra-cavity laser is shown in Fig. 5 [5, 6]. The system contains an erbium-doped fiber amplifier (EDFA), an Fabry-Perot (F-P) type tunable optical filter, a gas cell, a photo-detector, and an isolator. The EDFA is pumped by a $980 \mathrm{~nm}$ pump laser via a 980/1550 WDM coupler. The gas cell with a reflector is coupled into the main cavity by a circulator after being selected by an optical switch. The photo-detector behind a 50:50 coupler can monitor the output signal. The photo-detector is sampled by analogue-to-digital converter (ADC) on the computer controlled Labview card, which also generates the drive signal of tunable optical filter (TOF) from the digital-to-analogue converter (DAC). FBG array is used as wavelength references, which can be used for gas recognition.

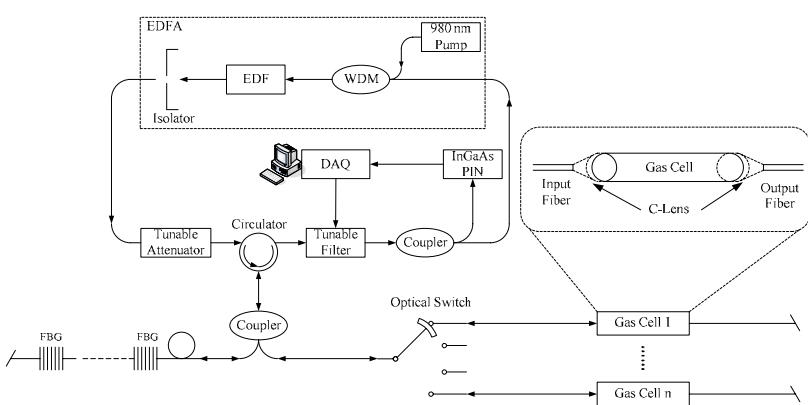

Fig. 5 Experimental configuration of the system based on intra-cavity laser.

The temporal behavior of the system can be described by rate equations as shown in [6]. Based on the temporal behavior model, the sensitivity enhancement factor of the system can be derived as [14]

$$
\frac{K}{\Delta \delta}=\frac{\Delta I}{I \Delta \delta} \propto \frac{A}{\delta(A-B \delta)}
$$

where $K$ is the absorption signal, $\Delta \delta$ is the absorption induced system loss variation, $A$ and $B$ are the constants determined by the system. It can be seen from the expression that the sensitivity is inversely proportional to $\delta(A-B \delta)$ and can therefore be enhanced by reducing the value of $\delta(A-B \delta)$. As the system loss $\delta$ can not be made too small, high sensitivity can only be achieved when the system is running close to the threshold $A-B \delta=0$.

When the system runs close to the threshold, the absorption lines of the detected gas can be obtained and used for calibration and detection with high sensitivity. The positions of the absorption lines in the spectra can be recognized by peak detection of the second derivative curve. And the concentration of the gas is proportional to the amplitude of Lorentzian fit spectrum. So the concentration of the detected gas can be calibrated and detected with the absorption spectra. The sensitivity of the system can be enhanced by taking the state of the system close to the threshold.

When the gas cell is filled with $1 \%$ acetylene concentration, the relationship between the absorption signal and the system attenuation is shown in Fig. 6. The maximum of the absorption 
signal is $7.47 \mathrm{~dB}$, corresponding to an enhancement of 37.4 times over a single-pass absorption loss of only $0.2 \mathrm{~dB}$.

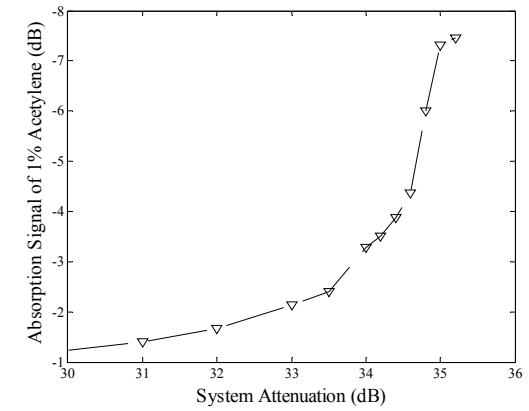

Fig. 6 Relationship between absorption signal and system attenuation.

The system attenuation of the system was chosen to $35 \mathrm{~dB}$. Under this condition, the relationship between the absorption signal and the concentration of acetylene is shown in Fig. 7. The responsibility is $-7.687 \pm 10^{-4} \mathrm{~dB} / \mathrm{ppm}$, with error less than $143 \mathrm{ppm}$. This result can be used to realize acetylene gas detection with high sensitivity.

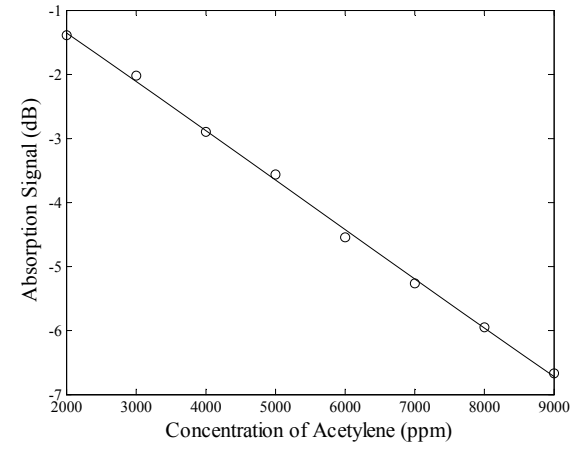

Fig. 7 Relationship between absorption signal and concentration of acetylene.

By using wavelength sweep technique and average algorithm, the sensitivity of the system can be less than $100 \mathrm{ppm}$. Combining wavelength sweep technique and wavelength modulation technique, the detection sensitivity of acetylene can be limited less than $75 \mathrm{ppm}$, which is only $0.3 \%$ of acetylene lower explosive limit. And gas type recognition can also be realized at the same time.

\section{Distributed stress sensing based on PMF}

In stress-induced PMF, polarization mode coupling will caused by a range of environment parameters, such as external transverse forces [7], distributed stress [8], position, and strain [9]. The coupling coefficient has a definite relationship with these parameters. Distributed fiber optics sensors employing white-light interferometry (WLI) based on polarization mode coupling in PMF are promising methods for their high spatial resolution and wide dynamic range, and it is insensitive to optical power fluctuation [10].

The sensors setup is shown in Fig. 8. The sensors adopt a simple scanning Michelson interferometer to compensate the OPD of two polarization eignemode to form the interferograms, and the intensity of the interferograms can be expressed as

$$
\begin{aligned}
I_{\text {out }} & =I_{0}\left\{1+\exp \left[-\left(\frac{d}{L_{\mathrm{co}}}\right)^{2}\right] \cos \left(k_{0} d\right)\right. \\
& \left.+\sqrt{h-h^{2}} \exp \left(-\frac{L_{\mathrm{co}}^{2} d^{2}}{2}\right) \cos \left(\Delta \beta l-k_{0} d\right)\right\}
\end{aligned}
$$

where $I_{0}$ is the intensity of light source, $d$ is the OPD of two mirrors, $k_{0}$ is the wavelength number in vacuum, $L_{\mathrm{co}}$ is the coherence length of light source, $h$ is the coupling strength of the coupling point in $\mathrm{PMF}, \Delta \beta$ is the propagating constant difference of two eignemodes, and $l$ is the length from the coupling point to the output end face of PMF. The magnitude and location of the stress can be given out by $h$ and $l$, respectively. Distributed measurement can achieved if several coupling points in the PMF. The position of the coupling points and the magnitude of the stress can be obtained by the interferograms.

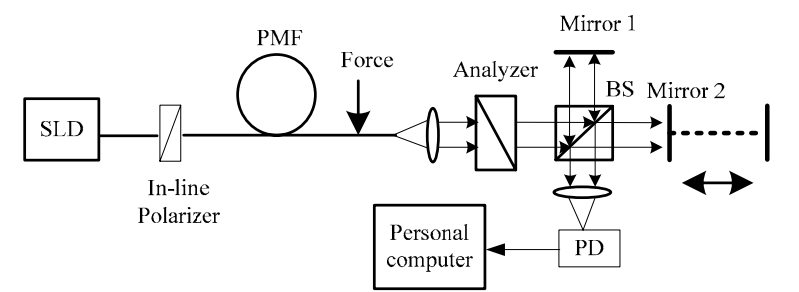

Fig. 8 Scheme diagram of PMFs sensors: SLD(superluminescent diode), BS(Beam splitter), PD(Photodiode).

From Fig. 9 we can know that the position or 
displacement of the coupling points can be obtained from the interferogram directly. If we ignore the magnitude of the coupling coefficient, the sensors can be used to position or displacement sensing which have a high spatial resolution and insensitive to electromagnetically interfere. The polarization maintaining devices can be also being tested to assure there are any coupling points in these devices intrinsic. If sensing heads that its character can be changed by any other environment parameters such as temperature and magnetic field are applied to PMF, the sensors can also be used to measure these parameters.

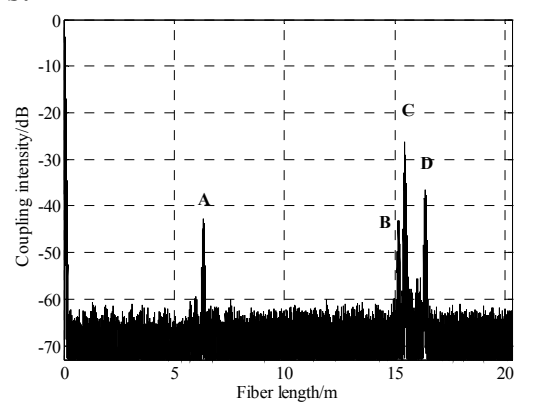

Fig. 9 Measured result of distributed sensoring.

\section{Prospect of current research on fiber intelligent sensing network}

Taking the intelligent sensing as the characteristic of the research of new generation optical fiber sensing network and the essential component will be the development direction of optical fiber sensing technology. Taking "exploring the new principle and structure of the new generation optical fiber sensors, resolving the new optical fiber microstructure sensing mechanism, breaking through the bottleneck of optical fiber nonlinear scattering effect, expanding the topology structure of the optical fiber intelligence sensing network, and laying the foundation for engineering applications" as the purport, the project "Fundamental Study of New Generation Fiber Intelligent Sensing Network and Key Devices", supported by National Basic Research Program of China (973 Program), complies with the huge social demand positively, vigorously carries out the basic science research of optical fiber sensing technology, and will enhance the optical fiber sensing technology entirely from structure, devices, technology, and sensing network.

The main purpose of the project is making the important breakthrough in the optical fiber intelligence sensing network, the essential devices, and basic research aspect. And there are four research aspects and six tasks in this project. We will focus on the research of optical microfluid based biology and chemistry optical fiber sensing mechanism, and new sensing devices and intelligent sensing mechanism. Discrete optical fiber intelligence sensing network will be developed and established. Now we are working on optical microfluid biology sensing and gas pressure detection research.

\section{Conclusions}

Tianjin University has engaged in the research of fiber sensing technologies for many years. We developed a fiber-optic temperature sensor based on the interference of selective higher-order modes in circular optical fiber, with temperature sensitivity of $15 \mathrm{pm} /{ }^{\circ} \mathrm{C}$ in the range of $100{ }^{\circ} \mathrm{C}$ to $750{ }^{\circ} \mathrm{C}$. Parallel demodulation for EFPI and FBG sensors is realized based on white light interference, with EFPI measurement precision of $0.81 \mu \mathrm{m}$ and FBG wavelength measurement precision of $14.4 \mathrm{pm}$. Concentration detection of acetylene is realized using intra-cavity fiber laser spectroscopy with sensitivity less than $100 \mathrm{ppm}$. And distributed position or displacement sensing was realized by using PMF. Our main work will be focused on optical microfluid biology sensing and gas pressure detection research.

\section{Acknowledgment}

This work was supported by National Basic Research Program of China (973 Program) under Grant No. 2010CB327800. 


\section{References}

[1] E. B. Li, X. L. Wang, and C. Zhang, "Fiber-optic temperature sensor based on interference of selective higher-order modes," Applied Physics Letters, vol. 89, no. 9, pp. 091119, 2006.

[2] E. B. Li and G. D. Peng, "Wavelength-encoded fiber-optic temperature sensor with ultra-high sensitivity," Optics Communications, vol. 281, no. 23, pp. 5768-5770, 2008.

[3] J. F. Jiang, T. G. Liu, Y. M. Zhang, L. N. Liu, Y. Zha, F. Zhang, Y. X. Wang, and P. Long, "Development of a parallel demodulation system used for extrinsic Fabry-Perot interferometer and fiber Bragg grating sensors," Applied Optics, vol. 45, no. 3, pp. 528-535, 2006.

[4] J. F. Jiang, T. G. Liu, Y. M. Zhang, L. N. Liu, Y. Zha, F. Zhang, Y. X. Wang, and P. Long, "Parallel demodulation system and signal processing method for extrinsic Fabry-Perot interferometer and fiber Bragg grating sensors," Optics Letters, vol. 30, no. 6, pp. 606, 2005.

[5] K. Liu, W. C. Jing, G. D. Peng, J. Z. Zhang, Y. Wang, T. G. Liu, D. G. Jia, H. X. Zhang, and Y. M. Zhang, "Wavelength Sweep of Intracavity Fiber Laser for Low Concentration Gas Detection," IEEE Photonics Technology Letters, vol. 20, no. 18, pp. 1515-1517, 2008.

[6] K. Liu, T. G. Liu, G. D. Peng, J. F. Jiang, H. X. Zhang, D. G. Jia, Y. Wang, W. C. Jing, and Y. M. Zhang, "Theoretical investigation of an optical fiber amplifier loop for intra-cavity and ring-down cavity gas sensing," Sensors and Actuators B: Chemical, vol. 146, no. 1, pp. 116-121, 2010.

[7] T. H. Xu, W. C. Jing, H. X. Zhang, K. Liu, D. G. Jia, and Y. M. Zhang, "Influence of birefringence dispersion on a distributed stress sensor using birefringence optical fiber," Optical Fiber Technology, vol. 15, no. 1, pp. 83-89, 2009.

[8] W. C. Jing, Y. M. Zhang, G. Zhou, H. X. Zhang, Z. H. $\mathrm{Li}$, and X. M. Man, "Rotation angle optimization of the polarization eigenmodes for detection of weak mode coupling in birefringent waveguides," Optics Express, vol. 10, no. 18, pp. 972-977, 2002.

[9] H. X. Zhang, T. H. Xu, D.G. Jia, W. C. Jing, K. Liu, and Y. M. Zhang, "Effects of angular misalignment in interferometric detection of distributed polarization coupling," Measurement Science and Technology, vol. 20, no. 9, pp. 095112, 2009.

[10] W. C. Jing, Y. M. Zhang, G. Zhou, F. Tang, and H. F. $\mathrm{Li}$, "Measurement accuracy improvement with PZT scanning for detection of DPC in Hi-Bi fibers," Optics Express, vol. 10, no. 15, pp. 685-690, 2002.

[11] K. O. Hill and G. Meltz, "Fiber Bragg Grating Technology Fundamentals and Overview," Journal of Lightwave Technology, vol. 15, no. 8, pp. 1263-1276, 1997.

[12] A. D. Kersey, M. A. Davis, H. J. Patrick, M. LeBlanc, K. P. Koo, C. G. Aksins, M. A. Putnam, and E. J. Frinebele, "Fiber Grating Sensors," Journal of Lightwave Technology, vol. 15, no. 8, pp. 1442-1463, 1997.

[13] M. Hart, D. G. Vass, and M. L. Begbie, "Fast surface profiling by spectral analysis of white-light interferogram with Fourier transform spectroscopy," Applied Optics, vol. 37, no. 10, pp. 1764-1769, 1998.

[14] Y. Zhang, M. Zhang, and W. Jin, "Sensitivity enhancement in erbium-doped fiber laser intra-cavity absorption sensor," Sensors and Actuators A, vol. 104, no. 1, pp. 183-187, 2003. 